Pathophysiology of Haemostasis and Thrombosis
Pathophysiol Haemost Thromb 2003/04;33:214-220

DOI: $10.1159 / 000081511$
Received: March 31, 2004

Accepted after revision: August 4, 2004

\title{
Alterations of the Global Haemostatic Function Test 'Resonance Thrombography' in Spontaneously Traumatised Dogs
}

\author{
R. Mischke \\ Small Animal Clinic, Hannover School of Veterinary Medicine, Hannover, Germany
}

\section{Key Words}

Trauma $\cdot$ Resonance thrombography

\begin{abstract}
Samples taken from 30 dogs with spontaneously acute trauma were investigated with two different resonance thrombographs (instrument 2: 28 dogs), a global method for examination of the haemostatic system. Reaction time of the resonance thrombogram (RTG-r), fibrin generation time (RTG-f), fibrin amplitude (RTG-F) and parameters of platelet function [amplitude of the platelet leg (RTG-P) and descending time of the platelet leg (RTG-p)] were evaluated statistically and compared with a normal control as well as with the results of individual components of the haemostatic system and other screening tests. Comparison of the results of the RTG of dogs suffering from trauma with the normal control revealed differences only for RTG-r, which was longer when measured with instrument 1 and lower RTG-F values measured with both instruments. Depending on the RTG parameter and instrument, only 1-6 samples showed values outside the reference ranges. Only 4 out of 26 cases (resonance thrombograph 2: 2 out of 24 cases) with mild to moderate deficiency in individual coagulation factor activity showed a prolongation of RTG-r which was significantly less sensitive than activated partial thromboplastin time ( $p<0.001)$. Of the 26 samples having mainly mildly to moderately increased
\end{abstract}

\section{KARGER}

Fax +41613061234

E-Mail karger@karger.ch

www.karger.com

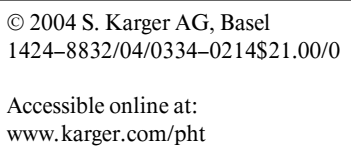

fibrin(ogen) degradation product (FDP) levels (median: $39 \mu \mathrm{g} / \mathrm{ml}$, range: $2-90 \mu \mathrm{g} / \mathrm{ml}$, reference values: $<18 \mu \mathrm{g} /$ $\mathrm{ml}$ ) only between 1 and 5 showed abnormalities of the parameters RTG-f and/or RTG-F. The results of this study show in particular the limited sensitivity of the method for detecting individual coagulation factor deficiencies and minimal to moderate increases of FDP concentrations. Remarkable alterations of fibrin generation as measured by the resonance thrombograph are a rare condition in dogs with acute accidental trauma.

Copyright $\odot 2004$ S. Karger AG, Basel

\section{Introduction}

Resonance thrombography was introduced by Hartert [1-3] in 1977 as a global method for haemostasis diagnostics. This dynamic procedure allows ex vivo observation of the haemostatic process (i.e., velocity, quantity, and quality of clot formation), including occasional hyperfibrin(ogen)olysis with the help of a graph (resonance thrombogram, RTG). As a further development of thrombelastography, resonance thrombography, in particular, allows for the differentiation between the effect of fibrin formation and platelet activity during clot formation. The principle of measurement is based, unlike thrombelastography, not on the elasticity of the clot but instead on the fibrin elasticity-induced resonance phenomenon. It seems to be especially valuable for detecting abnormal 
Table 1. Results of different tests of the haemostatic system in 30 dogs suffering from spontaneous trauma in the previous $24 \mathrm{~h}$

\begin{tabular}{|c|c|c|c|c|c|c|}
\hline \multirow[t]{2}{*}{ Parameter } & \multirow{2}{*}{$\begin{array}{l}\text { Reference } \\
\text { values }^{\mathrm{a}}\end{array}$} & \multirow{2}{*}{$\begin{array}{l}\text { Median } \\
\text { (range) }\end{array}$} & \multirow{2}{*}{$\begin{array}{l}\text { MWT }^{\text {b }} \\
\text { (p value) }\end{array}$} & \multicolumn{3}{|l|}{$\mathrm{n}^{\mathrm{c}}$} \\
\hline & & & & $<$ & $=$ & $>$ \\
\hline Platelet count, $10^{3} / \mu 1$ & $155-454$ & $237(99-338)$ & 0.0093 & 3 & 27 & 0 \\
\hline APTT reagent $1, \mathrm{~s}$ & $14.1-18.6$ & $19.5(14.7-33.8)$ & $<0.0001$ & 0 & 10 & 20 \\
\hline APTT reagent $2, \mathrm{~s}$ & $10.5-13.3$ & $13.1(11.3-23.5)$ & $<0.0001$ & 0 & 17 & 13 \\
\hline Thrombin time, s & $14.9-21.5$ & $15.5(12.0-19.5)$ & $<0.0001$ & 13 & 17 & 0 \\
\hline Factor V activity, \% & $75-158$ & $53(12-115)$ & $<0.0001$ & 23 & 7 & 0 \\
\hline Fibrinogen kinetic, g/l & $1.17-3.09$ & $2.33(0.55-4.31)$ & 0.0113 & 2 & 19 & 9 \\
\hline FDP concentration, $\mu \mathrm{g} / \mathrm{ml}$ & $<18$ & $39(2-90)$ & $<0.0001$ & 0 & 4 & 26 \\
\hline
\end{tabular}

a $2.5-97.5 \%$ quantile (FDP: $95 \%$ quantile) of the results of $\geq 102$ healthy dogs.

b Statistical comparison with the results of healthy dogs using Mann-Whitney test (MWT).

c Number $(\mathrm{n})$ of results below $(<)$, within $(=)$ and above $(>)$ reference values.

APTT $=$ Activated partial thromboplastin time; FDP $=$ fibrin $($ ogen $)$ degradation products.

fibrin formation, such as in disseminated intravascular coagulation/hyperfibrin(ogen)olysis complex [2, 4, 5]. Resonance thrombography is user-friendly and can be used as a bedside test to monitor haemostatic function.

Systematic investigations of alterations of RTG in different diseases including measurements of the sensitivity are rare even in human medicine [6,7]. Therefore, in this paper the results of our own study are published which are based on the systematic investigation of spontaneously traumatised dogs using two different resonance thrombographs.

\section{Materials and Methods}

\section{Animals}

This study included 30 unselected dogs (resonance thrombograph 2: $28 \mathrm{dogs}$ ) that presented to the Small Animal Clinic, Hannover School of Veterinary Medicine with clinical signs of shock due to accidental injury by a car. Blood samples were taken at the earliest possible time (and before treatment to avoid dilution by fluid therapy). Dogs were only included in this study if the accident had taken place during the previous $24 \mathrm{~h}$. No other exclusion criteria were considered.

Nineteen of the patients had fractures, including 4 dogs with multiple fractures. In 11 of these 19 dogs clinical examination and radiographs indicated additional thoracic trauma (e.g. lung haemorrhage, pneumothorax). In one further dog, this was associated with a diaphragmatic rupture and in another with a rupture of the spleen. Of the remaining 11 dogs (without fractures), 5 showed thoracic trauma, 1 had a diaphragmatic rupture, 1 had a diaphragmatic rupture and abdominal trauma, 2 had tail avulsion, 1 a hip luxation and 1 dog a tooth fracture and claw avulsion.

The results of the selected parameters which are related to the RTG results are summarised in table 1 . The most significant reduc- tion of an individual coagulation factor was found in factor $\mathrm{V}$ activity. In addition, activities of the residual coagulation factors were reduced in 8-23 of the patients. In total, 26 patients showed reduced activity of at least one of the activated partial thromboplastin time (APTT)-related clotting factors (intrinsic and common pathway). However, individual coagulation factor activities of $<30 \%$ of normal were found in only 5 patients. Detailed results of the haemostatic changes in these patients as well as the methods used to perform these tests have been published elsewhere [8].

For each parameter, measurements were done of at least 102 (up to 487 , dependent on the parameter) healthy adult dogs, all $\geq 1$ year of age, both males and females, and of different breeds. These were used for the calculation of reference values as well as a control group for statistical comparison.

\section{Collection of Sample Material for RTG}

Blood samples for resonance thrombography measurements were obtained from a cephalic or saphenous vein using sterile disposable needles $(1.1 \times 30 \mathrm{~mm})$ and using only minimal pressure to raise the vein. Blood was collected into plastic tubes containing one part $(1 \mathrm{ml})$ $0.11 \mathrm{ml} / \mathrm{l}$ sodium citrate solution for nine parts $(9 \mathrm{ml})$ of blood and immediately mixed by careful rocking.

\section{Resonance Thrombography}

Principle

In resonance thrombography, a metal pendulum inserted into a cuvette with recalcified citrated blood oscillates in an orbital pattern. This initially creates a blood flow analogous to that in the blood vessel. The oscillation radius of the pendulum is continually registered in the form of a graph (RTG) (fig. 1). The elasticity of the fibrin fibres that are forming leads to an increase in the drive frequency of the oscillation of the pendulum to the resonance level of the exciting oscillation and, thereby, increases the oscillation radius of the pendulum (rise in the curve, fibrin leg). The subsequent decrease in the radius of the pendulum oscillation (drop in the curve, platelet leg) reflects the contraction tension caused by the thrombocytes within the clot. 
Fig. 1. Schematic diagram illustrating the different RTG parameters.

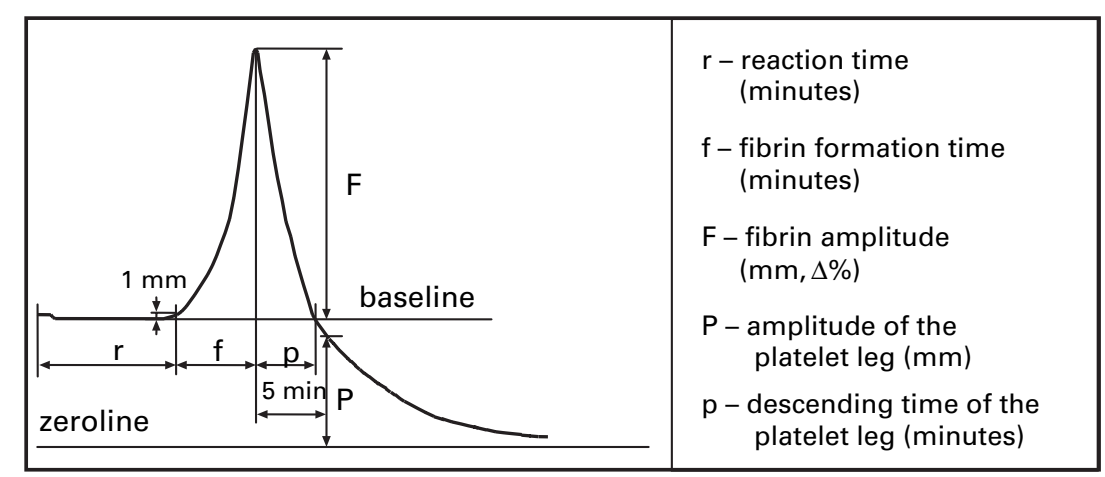

Performance/Test Prescription

The measurements were done with two instruments: (1) resonance thrombograph RTG-S 801 (instrument 1; Von Hoerner and Sulger Electronic $\mathrm{GmbH}$, Schwetzingen, Germany) as well as (2) Orbitometer ROM-4 (Trinity Biotech GmbH, Lemgo, Germany).

The measurements using instrument 1 were carried out following the original test instructions given for human blood [3]. $200 \mu 1$ citrated blood were filled into the RTG cuvette and prewarmed for $2 \mathrm{~min}$ at $37^{\circ} \mathrm{C}$ in the resonance thrombograph. Then recalcification was done by adding $200 \mu 116.7 \mathrm{mmol} / \mathrm{CaCl}_{2}$. Simultaneously, the registration was started. After mixing the testing solutions with a plastic stirring spatula, the metal pendulum taken from the heating device of the resonance thrombograph was inserted into the instrument and moved down into the cuvette containing recalcified blood. For this instrument, the evaluation of the RTG had to be effected manually by means of a special template. The characteristic parameters of RTG are illustrated in figure 1: reaction time (RTG-r), fibrin formation time (RTG-f), fibrin amplitude (RTG-F), amplitude of the platelet leg (RTG-P), and descending time of the platelet leg (RTG-p).

In preliminary, unpublished investigations the test instruction for measuring canine blood with instrument 2 was optimised as follows: prewarming of $300 \mu \mathrm{l}$ citrated blood in the cuvette in the heating device for $2 \mathrm{~min}$. Then, recalcification with $100 \mu 125 \mathrm{mmol} / \mathrm{l} \mathrm{CaCl}_{2}$ solution. Although values were generated automatically, only RTG-r, RTG-f, RTG-F and RTG-p were given by this machine.

\section{Statistical Analysis}

Reference values were defined by using control dogs on the basis of 2.5 and $97.5 \%$ quantiles. Data of the dogs suffering from trauma were presented as minimum, $25 \%$ quantile, $50 \%$ quantile (median), $75 \%$ quantile and maximum. The Mann-Whitney test was used for comparison of the results of the traumatised dogs with the control group due to the non-standard normally distributed data. Values of $\mathrm{p}<0.05$ were considered as significant. Sensitivity, specificity, and positive and negative predictive values for individual coagulation factor deficiencies were calculated for RTG-r and APTT. The number of the positive (and negative) results of the different parameters were compared using Fisher's exact test. Spearman's rank correlation coefficient $\left(\mathrm{r}_{\mathrm{s}}\right)$ was calculated for the relationship between the results of the RTG-r and APTT.

\section{Results}

Comparison of the results of RTG of dogs suffering from trauma with normal controls revealed that the only significant differences in dogs following trauma were a longer RTG-r measured with instrument 1 and lower RTG-F values measured with both resonance thrombographs (fig. 2, 3). Most of the individual measurement values of the different RTG parameters in traumatised dogs were within reference ranges. Depending on the RTG parameter and instrument, 1-6 samples showed values outside the reference values.

Prolongation of RTG-r was found in only 4 out of 26 cases (machine 2: 2 out of 24 cases) with mainly mild to moderate deficiency in individual coagulation factor activity. Of the 5 plasma samples having at least one individual coagulation factor activity $<30 \%$ of normal only one led to an increased RTG-r (instrument 1; instrument 2: out of 4 tested). Therefore, RTG-r reflects the decrease in clotting factor activity of the intrinsic and common pathways with significantly less sensitivity compared to the APTT ( 20 or 13 abnormal results out of 30 depending on the reagent; $p<0.001$ ) (table 2). Associated with the low sensitivity of RTG-r for coagulation factor deficiencies, specificity and positive predictive values were high (between 0.75 and 1.0) and negative predictive values were low (instrument 1: 0.12; instrument 2: 0.16). Based on the sample material investigated, specificity and positive and negative predictive values did not differ significantly between RTG-r and APTT. The correlation between APTT and RTG-r was not significant ( $p>0.05)$, independent of the resonance thrombograph and the APTT reagent (resonance thrombograph 1/reagent 1: $\mathrm{r}_{\mathrm{s}}=$ 0.269 and reagent $2: r_{s}=0.104$; resonance thrombograph 2/reagent 1: $r_{s}=0.229$ and reagent $2: r_{s}=0.272$ ).

In all 9 samples with increased fibrinogen concentration RTG-f was within the reference range and in 3 of the 


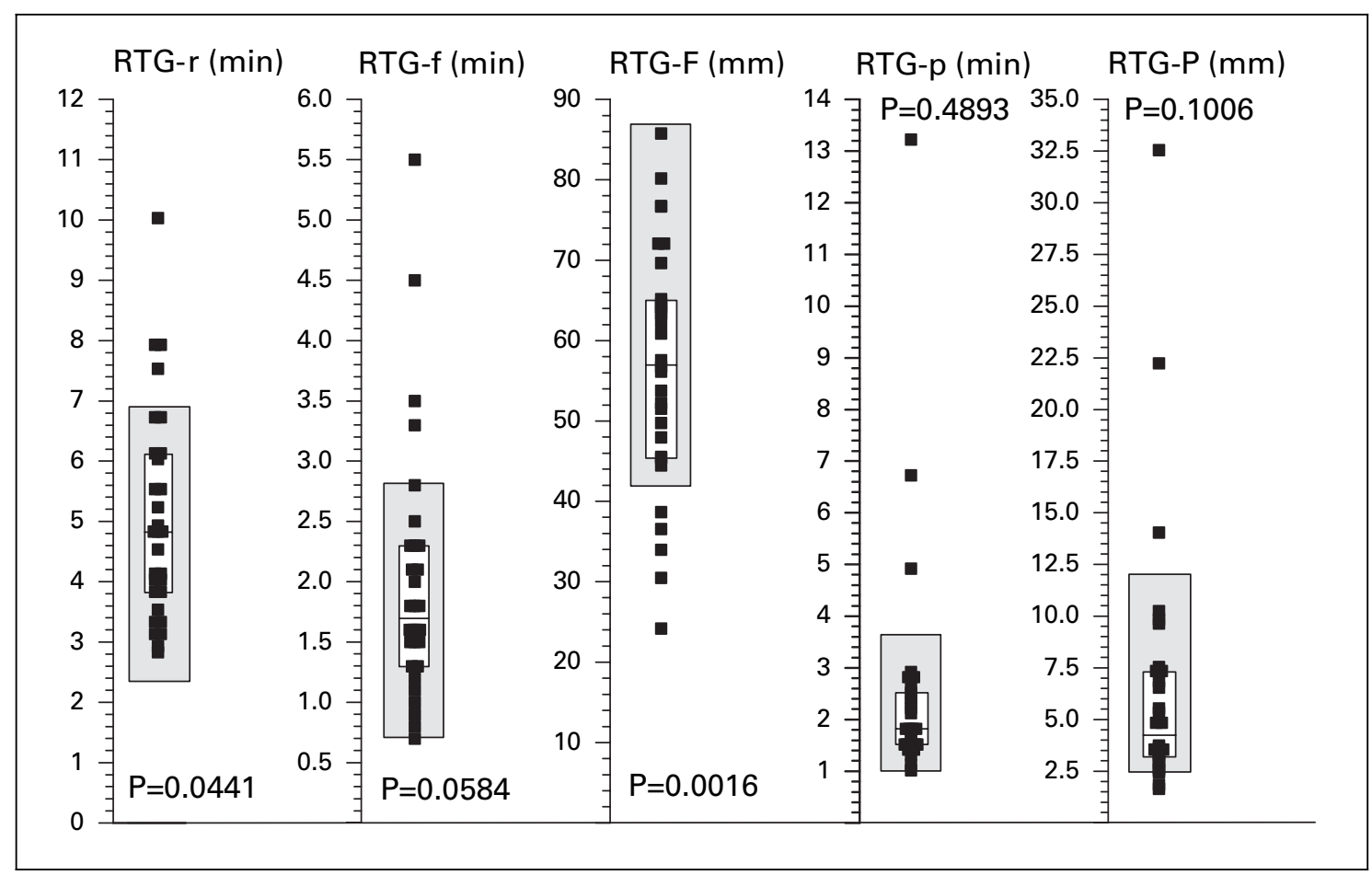

Fig. 2. Results of different parameters of the resonance thrombograph RTG-S 801 in 30 dogs suffering from spontaneous trauma in the previous $24 \mathrm{~h}$. The white box indicates the $25 \%$ quantile, median, and $75 \%$ quantile. The shaded box indicates the reference values based on the $2.5-97.5 \%$ quantile of the results of 452 healthy dogs. p values are derived from statistical comparison with the results of healthy dogs using Mann-Whitney test.

Fig. 3. Results of different parameters of the resonance thrombograph Orbitometer ROM-4 in 28 dogs suffering from spontaneous trauma in the previous $24 \mathrm{~h}$. The white box indicates the $25 \%$ quantile, median, and $75 \%$ quantile. The shaded box indicates the reference values based on the $2.5 \%-97.5 \%$ quantile of the results of 305 healthy dogs. p values are derived from statistical comparison with the results of healthy dogs using the Mann-Whitney test. a) = Increased values not detectable, because upper limit of reference values $>$ upper limit of measuring range.

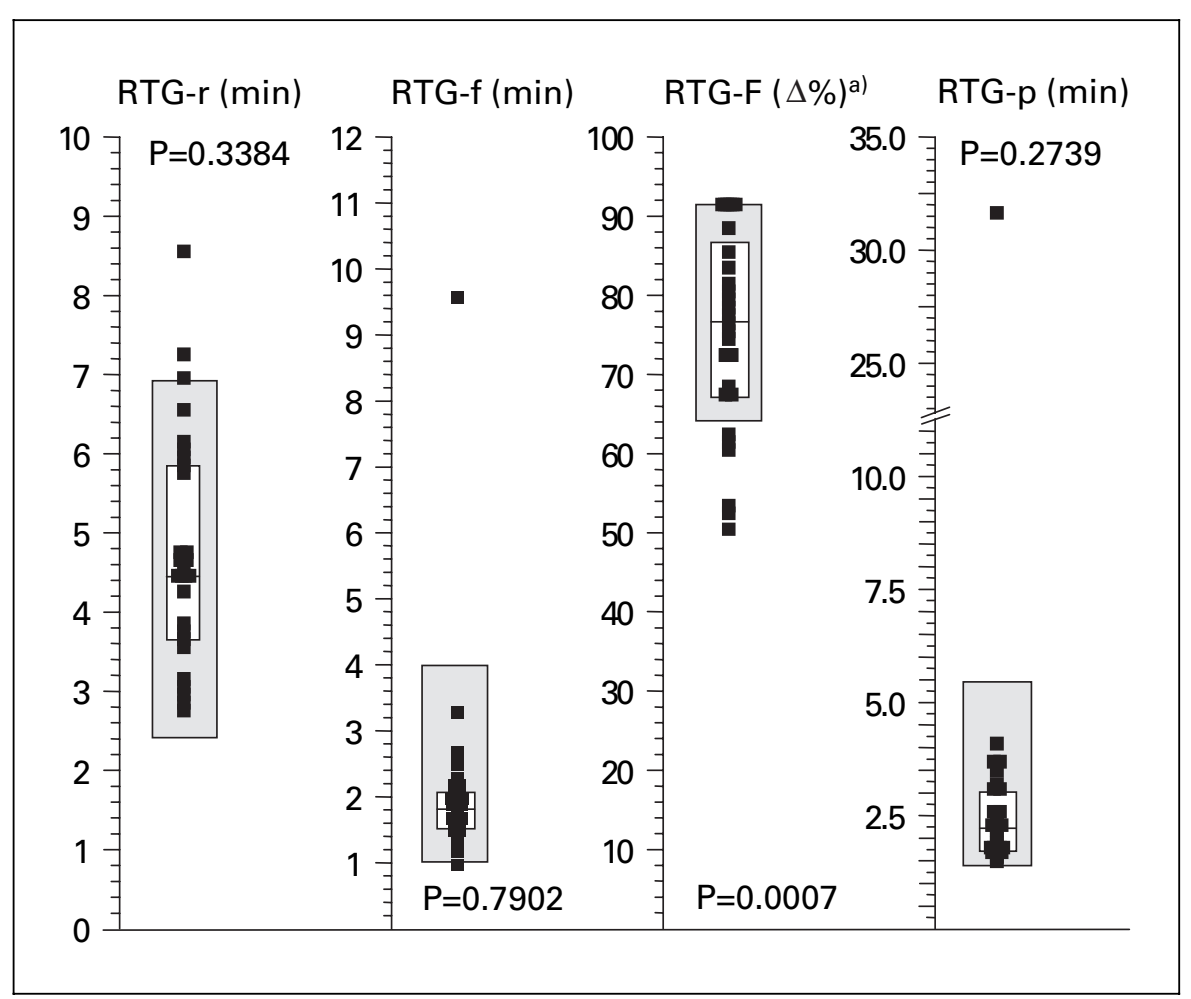

Pathophysiol Haemost Thromb 2003/04:33:214-220 
Table 2. Comparison of the sensitivity, specificity, positive and negative predictive values of the RTG-r (two different instruments) as well as of the APTT (two different reagents) to detect mild to moderate deficiencies of the APTTdependent coagulation factors found in $n=26$ (instrument $2: n=24$ ) out of 30 spontaneously traumatised dogs

\begin{tabular}{|c|c|c|c|c|}
\hline \multirow[t]{3}{*}{ Method } & \multicolumn{2}{|l|}{ RTG-r } & \multicolumn{2}{|l|}{ APTT } \\
\hline & machine RTG-S 801 & $\begin{array}{l}\text { machine Orbitometer } \\
\text { ROM-4 }\end{array}$ & reagent 1 & reagent 2 \\
\hline & A & B & $\mathrm{C}$ & $\mathrm{D}$ \\
\hline Sensitivity ${ }^{a}$ & $0.12(3 / 26)$ & $0.08(2 / 24)$ & $0.73(19 / 26)$ & $0.50(13 / 26)$ \\
\hline Specificity ${ }^{\mathrm{b}}$ & $0.75(3 / 4)$ & $1.00(4 / 4)$ & $0.75(3 / 4)$ & $1.00(4 / 4)$ \\
\hline Positive predictive value $\mathrm{e}^{\mathrm{b}}$ & $0.75(3 / 4)$ & $1.00(2 / 2)$ & $0.95(19 / 20)$ & $1.00(13 / 13)$ \\
\hline Negative predictive value ${ }^{b}$ & $0.12(3 / 26)$ & $0.16(4 / 26)$ & $0.30(3 / 10)$ & $0.24(4 / 17)$ \\
\hline
\end{tabular}

Significant differences (Fisher's exact test): ${ }^{a} \mathrm{~A} / \mathrm{C}=\mathrm{p}<0.0001, \mathrm{~A} / \mathrm{D}=\mathrm{p}=0.0033, \mathrm{~B} / \mathrm{C}=\mathrm{p}=0.0001, \mathrm{~B} / \mathrm{D}=$ $\mathrm{p}=0.0018 .{ }^{\mathrm{b}}$ Without significant difference. Figures in parentheses illustrate the original data for calculating the statistical values.

samples RTG-F was reduced, irrespective of the instrument used (fig. 4b). One single patient (P 442) suffering from a diaphragmatic rupture with a prolapse of the liver as well as abdominal trauma (splenic rupture, rupture of the kidney capsule) showed a remarkably reduced fibrinogen concentration $(0.55 \mathrm{~g} / \mathrm{l})$. This was associated with an abnormally wide curve pattern with both machines (fig. 4c). Despite this fact, RTG-F measured using instrument 1 was within the reference values. In another dog with a slightly reduced fibrinogen concentration $(0.93 \mathrm{~g} / \mathrm{l})$ all measurement values were within the reference range. Of the 26 samples having mildly to moderately increased fibrinogen degradation product (FDP) levels, dependent on the instrument and parameter, only 1-5 showed abnormalities of the RTG-f and/or RTG-F.

In one dog with minimally reduced platelet count $(134,000 / \mu 1)$ none of the RTG parameters showed an abnormal result. Two dogs showed slightly reduced platelet counts $(99,000 / \mu 1)$. In one of these dogs, where only the resonance thrombograph 1 was used, RTG-p and RTG-P showed abnormal results. In the other dog RTG-p values with both instruments and RTG-P (instrument 1) were abnormal as part of the deformed RTG curve due to a reduced fibrinogen concentration and increased FDP level (P 442, fig. 4c).

\section{Discussion}

Based on the frequent coagulation factor activity decreases present in the traumatised dogs due to decompensated consumption, the low number of abnormal RTG-r results, irrespective of the instrument used, indicates the low sensitivity of the RTG-r in detecting individual coagulation factor deficiencies. With regard to the mainly mild to moderate activity decrease of the coagulation factors this indicates the limits of a global screening test. It is a well-recognised phenomenon that activities of individual coagulation factors have to decline below $30 \%$ of normal to induce a significant alteration of haemostasis [9]. However, even coagulation factor activities below this level which are probably associated with a significantly increased clinical bleeding tendency were not accurately detected. This concurs with the results of a preliminary study by the author in which the accuracy of the reaction time of the RTG was investigated to detect a decrease in clotting factors VIII:C and IX in samples of dogs with haemophilia A and B. Consistent with the results of the present study only a low sensitivity was found, which was especially expressed by the fact that even some of the samples with a factor VIII:C activity below 5\% showed falsenegative results [10].

The fact that APTT is more sensitive reflects the additional factors which influence the measurement of a citrated blood sample using resonance thrombography (availability of platelet factor 3, i.e. adequate platelet count, haematocrit). This fact is also expressed in the low degree of correlation between RTG-r and APTT. The correlation coefficients calculated in the present study based on traumatised dogs are approximately in the same range as the correlation coefficient which was calculated based on 315 samples of 105 human beings who underwent cardiac surgery $(r=0.20, p<0.05$ [7]). Due to the low number of cases with normal individual coagulation factor activity (4 out of 28 or 30) calculations of specificity and negative predictive values have to be interpreted carefully. 
The great advantage of the resonance thrombograph is that it can be used as a bedside test and that it gives a global overview of different aspects of haemostasis $[1,7]$. It has been suggested that it may be a diagnostic tool to decide whether therapy with coagulation factors, fibrinogen and platelet concentrates is indicated and to monitor its effectiveness [2,7]. However, the results of the present study show that the resonance thrombograph is not useful in assessing the need for coagulation factor supplementation.

Despite the fact that the FDP concentration was elevated in 26 of the patients, only a small number of RTG results of the RTG-f as well as RTG-F were outside the reference ranges. These parameters illustrate the resonance phenomenon and, therefore, indicate the frequency of clot formation and stability of the clot. This is the main advantage of the RTG compared to clotting tests measuring only the time until formation of the first fibrin strands. Previous investigations based on the in vitro addition of canine fibrinogen fragments to the blood or plasma of healthy dogs have shown that RTG reacts more sensitively to the fibrin formation alterations induced by FDP [11] than e.g. the thrombin time [12]. FDP decreases fibrin stability by integration into the fibrin clot (especially FDP X) and indirectly by forming soluble complexes with fibrin monomers, leading to decreasing amounts of fibrin monomers available for fibrin clot formation. In addition, substrate competition with fibrinogen for the fibrinogen binding site of thrombin has to be considered as an anticoagulatory effect of FDP [13-15].

The preliminary studies based on in vitro addition of FDP have shown that concentrations of for example $\geq 60 \mu \mathrm{g} / \mathrm{ml}$ fibrinogen fragment $\mathrm{Y}, \geq 150 \mu \mathrm{g} / \mathrm{ml}$ fragment $\mathrm{X}$ or $\geq 170 \mu \mathrm{g} / \mathrm{ml}$ fragment $\mathrm{D}$ are required to cause alteration of the RTG (RTG-f) to the limit of the reference range [12]. Thus, the highest FDP levels which were found in traumatised dogs were around the (lower) detection limit of the method which explains the low number of abnormal RTG-f and RTG-F results. In dogs suffering from trauma the increased median fibrinogen concentration also has to be considered because high fibrinogen levels decrease the sensitivity of the assay to detect FDP. The accuracy of the serum-based test for estimating the FDP level which was used in our study has been questioned in the literature [16]. The main points of criticism are (1) a possible incomplete removal of the fibrinogen (false high results) and also (2) a possible incorporation of FDP into the clot leading to an underestimation of the real FDP concentration. However, results of recovery experiments have shown that this test measures most of the canine
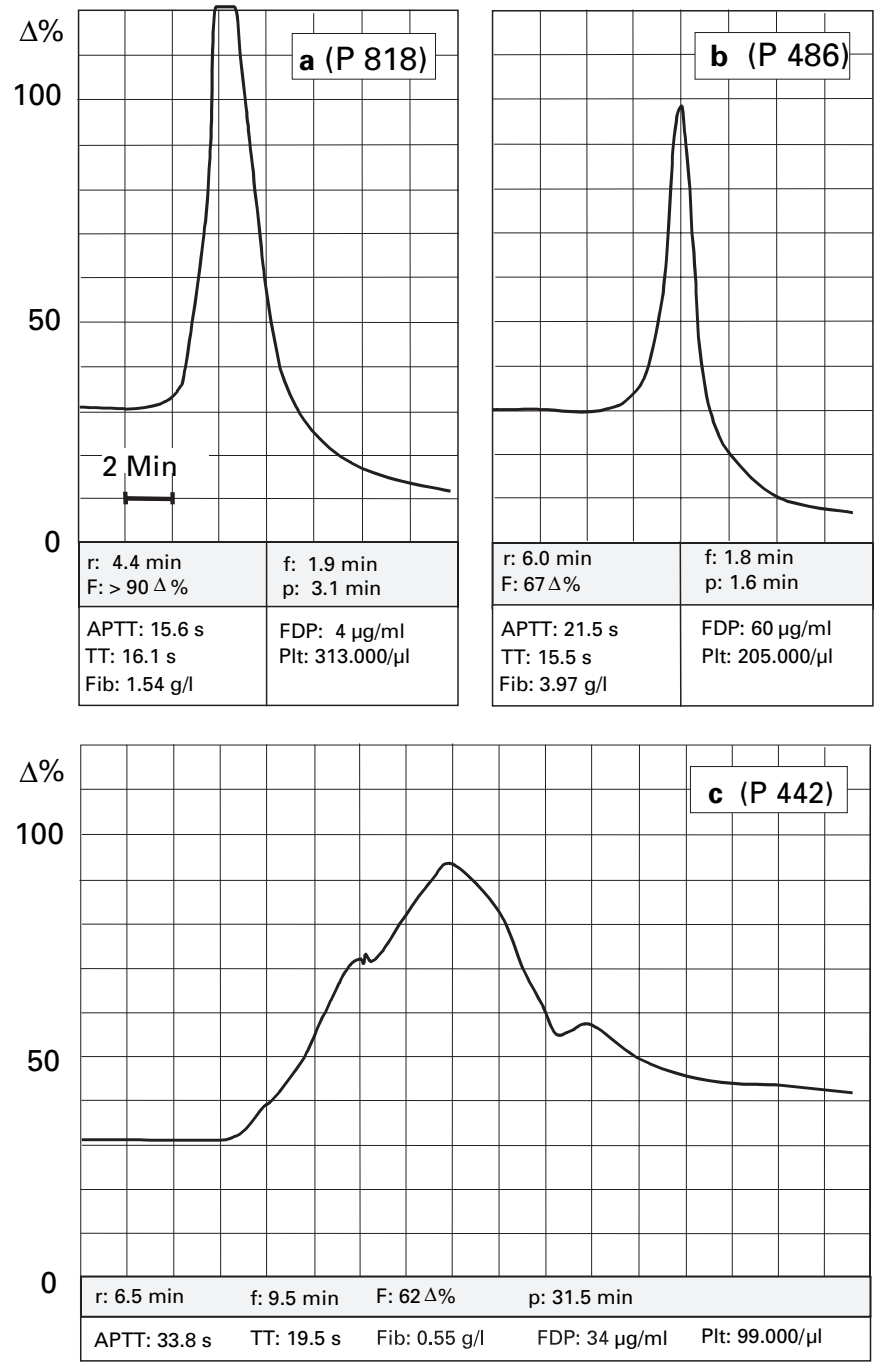

Fig. 4. RTG of three dogs suffering from trauma. a Normal finding. b Short, narrow curve associated with increased fibrinogen concentration. c Short, wide curve due to hypofibrinogenaemia and increased FDP level. TT = thrombin time; Fib = fibrinogen; $\mathrm{Plt}=$ platelet count.

FDP reliably with the exceptionof the earliest fragment X, which differs from fibrin monomers by the cleavage of diminutive parts from the craboxy-terminal end of the $\alpha$ chain in the D domains [17].

The fact that the most significant changes compared to normal dogs were found for RTG-F reflects, in part, the nonlinear relationship between fibrin stability and this parameter $[1,11]$. The number of samples with reduced RTG-F, therefore, includes samples with high fibrin stability (increased fibrinogen concentration) causing a short, narrow curve as well as samples with reduced fibrin 
stability causing a flat and broad curve. Consequently, nonselective sensitivity calculations for the RTG-F based on the whole number of samples with decreased values will lead to artificial results. This misinterpretation of individual parameters is avoided if, under normal clinical conditions, the interpretation is based on the whole RTG curve pattern. Assuming that resonance thrombography, based on its measurement principle, is a valuable test of fibrin clot stability [1] the low number of abnormal results indicates that the clinically relevant alteration of fibrin formation is a rare condition in spontaneously traumatised dogs.

When interpreting the low number of RTG alterations in dogs with increased fibrinogen concentration, it should be remembered that the maximum value which the acute phase reactant fibrinogen reached during the first $24 \mathrm{~h}$ after trauma exceeded the upper limit of the reference range only by 1.3 times. Additionally, it has to be considered that the parallel increase in FDP in the traumatised dogs should lead to a decreased sensitivity for the detection of increased fibrinogen concentration. In a previous study 35 RTGs out of 52 canine samples investigated with increased (but not further specified) fibrinogen concentrations showed abnormal RTG-F values [18]. Especially differences in the degree of the fibrinogen increase may explain this discrepancy.

Whereas a group of dogs suffering from trauma is particularly suitable for investigating the sensitivity of RTG to detect mild to moderate decreases of coagulation factors and increases in FDP, the low number of cases with a decreased fibrinogen concentration and thrombocytopenia did not allow for the accurate assessment of these alterations in our study.

In conclusion, the results of the present study show the limits of resonance thrombography as a global screening test of haemostasis for the detection of mainly mild to moderate changes of the haemostatic system present in dogs with trauma, especially individual coagulation factor deficiencies and increases of the FDP concentration. On the other hand, the results indicate that severe alterations of fibrin generation are a rare condition in traumatised dogs.

\section{References}

$>1$ Hartert H: Resonance-thrombography: Theoretical and practical elements. Biorheology 1981;18:693-701.

2 Hartert H: Differenzierung der Therapie von Gerinnungsstörungen durch Diagnostik mit dem Resonanzthrombographen. Therapiewoche 1983;33:4626-4634.

$\checkmark 3$ Hartert H: Biorheology in the practice of medicine: Resonance thrombography. Biorheology 1984:21:19-32.

4 Geiss V, Geiss B, Nolte I: Hämostasestörungen bei malignen Hämangioendotheliomen und Leukosen des Hundes - Aussagen der Resonanzthrombographie. Tierärztl Prax 1989; 5(suppl):68-71.

$>5$ Mischke R, Nolte I: Labordiagnostik und Differentialdiagnose der Verbrauchskoagulopathie beim Hund. Berl Münch Tierärztl Wochenschr 1992;105:401-410.

6 Anders O, Görss E-W, Ernst B, Konrad H: Erfassung des Faktor-XIII-Mangels bei akuter Leukämie mit der Resonanzthrombographie. Folia Haematol 1987;114:670-679.

7 Hinsenbrock K-P, Anders O, Ernst B, Neumann B, Förster J: Hämostaseologisches Monitoring in der Intensivtherapie: Untersuchungen mittels Resonanzthrombographie. Anaesthesiol Reanim 1989;14:219-225.
8 Mischke, R: Acute haemostatic changes in accidentally traumatised dogs. Vet $\mathrm{J}$, in press.

9 Proctor RR, Rapaport SI: The partial thromboplastin time with kaolin: A simple screening test for first stage plasma clotting factor deficiencies. Am J Clin Pathol 1961;36:212-219.

10 Mischke R: Sensitivity of reaction time of the resonance thrombogram for factor VIII:C and factor IX deficiencies in the blood of dogs with haemophilia A or B. Haemophilia 2000;6:575580.

11 Mischke R, Wolling H, Nolte I: Detection of anticoagulant activities of isolated canine fibrinogen degradation products $\mathrm{X}, \mathrm{Y}, \mathrm{D}$ and $\mathrm{E}$ using resonance thrombography. Blood Coagul Fibrinolysis 2004;15:81-88.

12 Mischke R, Wolling H: Influence of fibrinogen degradation products on thrombin time, activated partial thromboplastin time and prothrombin time of canine plasma. Haemostasis 2000;30:123-130.

13 Haverkate F, Timan G, Nieuwenhuizen W: Anticlotting properties of fragments D from human fibrinogen and fibrin. Eur J Clin Invest 1979;9:253-255.
14 Marder VJ, Shulman NR: High molecular weight derivatives of human fibrinogen produced by plasmin. II. Mechanism of their anticoagulant activity. J Biol Chem 1969;244: 2120-2124.

15 Nieuwenhuizen W, Voskuilen M, Hermanns J: Anticoagulant and calcium-binding properties of high molecular weight derivatives of human fibrinogen (plasmin fragments Y). Biochim Biophys Acta 1982;708:313-316.

16 Gaffney PJ, Perry MJ: Unreliability of current serum fibrin degradation product (FDP) assays. Thromb Haemost 1985;53:301-302.

17 Wolling H, Mischke R: Prüfung der Kreuzreaktivität und Sensitivität eines kommerziellen FDP-Latex-Agglutinationtests gegenüber reinen caninen Fibrin(ogen)spaltprodukten. Kleintierpraxis 1995;40:23-32.

18 Pause B: Vergleichende Untersuchung zur Gerinnungsdiagnostik mit dem Resonanzthrombographen und dem Thrombelastographen gemessen an der Thrombozytenzahl, dem Fibrinogengehalt, der Prothrombinzeit und der aktivierten partiellen Thromboplastinzeit bei klinisch gesunden und kranken Hunden, Katzen und Pferden; thesis University of Giessen, 1988. 